estimated 6 additional chlamydia positive partners would have been identified totalling 18 .

Discussion/Conclusion Only 1/3rd of our target group were offered, we calculate 54 new chlamydia positives (18\% of target) if offered to all. Kits costs $£ 1.30$, we distributed 415 costing $£ 539$. We identified 12 patients $(7+5)$ and estimate an extra 6 would have been found so each chlamydia positive costs $£ 30$ $£ 45$ (test kit only).

\section{P208 AUDIT OF THE MANAGEMENT OF HIV POSITIVE PREGNANT WOMEN IN A LOW PREVALENCE SETTING}

Kasey Redler*, Rebecca Hallgren, Rebecca Swingler.

Gloucestershire Hospitals NHS Foundation Trust, Gloucester, UK.

\subsection{6/sextrans-2016-052718.256}

Background/introduction HIV Maternal to child transmission (MTCT) has reduced from 25\% in 1993 to $0.57 \%$ in 2007 due to increased intervention in pregnancy. Compliance with BHIVA guidelines requires multidisciplinary care, which could be a challenge in areas with low HIV prevalence.

Aim(s)/objectives To audit the management of HIV positive pregnant women in a large district general hospital (DGH) against BHIVA guidelines.

Methods Retrospective audit of all HIV positive women giving birth at this DGH between September 2010 and October 2015.

Results 21 women identified. Diagnosis: 100\% screened for STI, hepatitis C, VZV and HIV. Treatment: $81 \%$ on HAART at start of pregnancy, $100 \%$ of the four women not on HAART were started on treatment during pregnancy. At start of pregnancy $61 \%(\mathrm{n}=13$ ) had a viral load $<50 \mathrm{RNA}$ copies $/ \mathrm{ml}$, by the end of pregnancy this increased to $86 \%(\mathrm{n}=18) .100 \%(\mathrm{n}=21)$ had MDT management. Delivery: 43\%: vaginal delivery. 38\%: elective Caesarean section. 19\% emergency Caesarean section. Post-partum care: $100 \%$ babies had post exposure prophylaxis started within 4 hours. No babies contracted HIV. 100\% babies exclusively bottle-fed. $57 \%$ mothers given carbegoline.

Discussion/conclusion There was good compliance with guidelines. All women received HAART and the MTCT rate was $0 \%$. $43 \%$ of women had a vaginal birth. Inclusion of the importance of carbergoline in departmental training may improve compliance in this area. In a low prevalence centre a specialist HIV antenatal clinic cannot be justified. The centre has introduced bimonthly MDT meetings to discuss these cases and these results suggest that communication and standards of care are high.

\section{P209 OUTCOMES OF PARTNER NOTIFICATION (PN) FOR SEX PARTNERS OF PEOPLE WITH CHLAMYDIA, MANAGED VIA THE ONLINE CHLAMYDIA PATHWAY.}

\footnotetext{
${ }^{1,2}$ Jo Gibbs* ${ }^{*}$ 'Pam Sonnenberg, ${ }^{3}$ Laura Tickle, ${ }^{1}$ Lorna Sutcliffe, ${ }^{4}$ Voula Gkatzidou, ${ }^{4}$ Kate Hone, ${ }^{2}$ Catherine Aicken, ${ }^{5} S$ Tariq Sadiq, ${ }^{1}$ Claudia Estcourt. ${ }^{1}$ Queen Mary University of London, London, UK; ${ }^{2}$ University College London, London, UK; ${ }^{3}$ Barts Sexual Health Centre, London, UK; ${ }^{4}$ Brunel University London, Uxbridge, UK; ${ }^{5}$ St George's, University of London, London, UK
}

\subsection{6/sextrans-2016-052718.257}

Background/introduction Within the $e \mathrm{STI}^{2}$ consortium, we conducted exploratory studies of an innovative Online Chlamydia Pathway (OCP: results service, automated clinical consultation, electronic prescription via community pharmacy, with telephone helpline support), which included optional online partner management.
Aim(s)/objectives To evaluate PN outcomes and sex partner uptake of online management.

Methods Participants: untreated GUM clinic attenders (two London services) and people tested through six London NCSP online postal testing areas: 21.07.14-13.3.15. The OCP offered index patients an SMS/email containing a unique code and link to forward to partners permitting them to access online care via OCP (patient referral). Outcomes were captured by OCP and index-reported at telephone follow-up (2 weeks post diagnosis)

Results Outcomes: 221 index patients consented to the study and $172(78 \%)$ were followed up by telephone (median age 23, $62 \%$ female). These 172 index patients reported 371 partners; $317 / 371(85 \%)$ were contactable and $256 / 317$ (81\%) of these were notified. Index patients reported 120/317 (38\%) as treated. Online outcomes: 154 index patients reached PN stage of OCP (some had already been routed to clinic). 94/154 (61\%) requested online partner access. They reported 280 partners: 28 went online; 19 received treatment at their chosen pharmacy; and 4 were treated elsewhere.

Discussion/conclusion 38\% of partners treated compares favourably with outcomes for routine PN within similar studies. Online management of sex partners through patient referral is feasible but uptake was low and most successful PN was achieved offline. Pathway optimisation could include anonymised sex-partner PN messaging and provision of partner STI self-sampling kits.

\section{P210 THE PREVALENCE OF CHLAMYDIA IN PREGNANT WOMEN COMPARED WITH NON-PREGNANT WOMEN IN A BUSY SEXUAL HEALTH CLINIC IN THE UK: MAKING THE CASE FOR SYSTEMATIC CHLAMYDIA SCREENING IN PREGNANCY?}

Cornelia Junghans*, Katherine Warren, Dale Coley, Eleanor Draeger. Lewisham and Greenwich NHS Trust, London, UK

\subsection{6/sextrans-2016-052718.258}

Background Chlamydia trachomatis (CT) is the commonest STI in the UK with high prevalence in pregnancy. CT testing is not

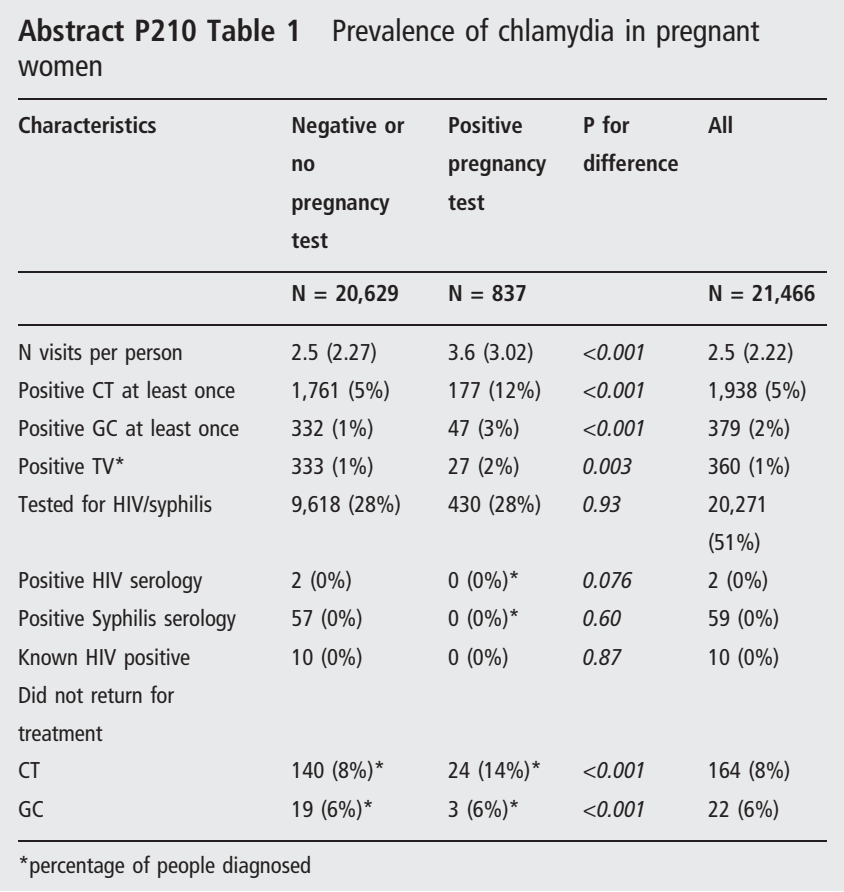


routinely recommended for pregnant women in the UK despite adverse effects on pregnancy, neonate and mother. Countries with recommended CT screening in pregnancy have good results despite suboptimal uptake.

Aim(s) To determine the prevalence of CT and treatment uptake in pregnant and non-pregnant women who have attended a large, busy urban integrated sexual health service.

Methods Retrospective observational cohort study of all patient encounters with women of child bearing age (15 to 49) between 2012 and 2015 who were tested for CT. Women with a positive pregnancy and STI testing 2 weeks prior and 9 months post STI test were included $(n=837)$. Women with terminations were excluded. Socio-demographic factors and clinical factors were compared to 20,629 women who had a CT test but no pregnancy.

Results: Pregnant women were on average younger (25 vs 28) and had higher CT prevalence across all age groups (12 vs 5\%), peaking between 15 and 25 years (21 vs 10\%). Being pregnant doubled the odds of having CT after controlling for age (adjusted OR 1.96; CI 1.64-2.30).

Discussion The national opportunistic screening programme is deemed to cover CT detection in an age group where both STIs and pregnancy rates are high. However, the results of our study support additional targeted CT screening during pregnancy, particularly in the youngest age group.

\section{P211 BASHH ON TWITTER: SOCIAL MEDIA FOR SEXUAL HEALTH}

${ }^{1}$ Adele Wolujewicz*, ${ }^{1}$ Mark Lawton, ${ }^{2}$ Nick Theobald, ${ }^{1}$ Martyn Wood. ${ }^{1}$ Royal Liverpool University Hospital, Liverpool, UK; ${ }^{2} B A S H H$, Macclesfield, UK

10.1136/sextrans-2016-052718.259

Background/introduction Twitter is a free, online social networking service through which users can read and send brief messages of up to 140-characters called “Tweets.” BASHH (@BASHH_UK) currently has 3479 followers on Twitter. These include professionals working in sexual health, sexual health services with their own Twitter accounts and members of the public. Twitter disseminates messages instantly and can influence a wide audience. It can be used to keep health professionals and patients informed about relevant research and headline news. For example, recent Twitter themes have included discussions about PrEP and gonorrhoea resistance. Twitter is also used for sharing practical information such as conference deadlines.

Aim(s)/objectives To evaluate the reach of BASHH on Twitter by reporting BASHH's Twitter activity.

Methods Using Twitter Analytics, data was obtained on the number of Tweets and Tweet Impressions for each of the last 4 months. Tweet Impressions are counted as the number of times each Tweet is seen. The number of visits to BASHH's Twitter profile, mentions by other Twitter users and number of new followers are also recorded. This is presented below:

Results

\begin{tabular}{|c|c|c|c|c|c|}
\hline Month & Tweets & Tweet Impressions & Profile Visits & Mentions & New followers \\
\hline November 2015 & 18 & 19200 & 1122 & 105 & 120 \\
\hline December 2015 & 31 & 36600 & 1548 & 83 & 67 \\
\hline January 2016 & 49 & 45500 & 2398 & 222 & 120 \\
\hline February 2016 & 33 & 28100 & 2394 & 125 & 108 \\
\hline
\end{tabular}

Discussion/conclusion BASHH's Twitter activity is reaching a large audience and increasing. All individuals working in sexual health and HIV should consider using Twitter to support wider engagement with their professional network.

\section{P212 WHICH PATIENTS MAY BE ELIGIBLE FOR PRE-EXPOSURE PROPHYLAXIS (PREP) FOR HIV?}

Helen Bradshaw, Laura Cunningham, Rachel Drayton, Nicola Lomax*. Cardiff and Vale University Healthboard, Cardiff, UK

\subsection{6/sextrans-2016-052718.260}

Background/introduction HIV diagnoses remain high among men who have sex with men (MSM). Recent studies show promising results with reducing MSM HIV transmission. The PROUD and IPERGAY studies, using daily or 'on demand' Truvada as PrEP respectively, showed an 86\% HIV transmission reduction. Clinical guidance and funding arrangements for PrEP are in development. We assessed PrEP eligibility amongst MSM attending our sexual health service.

Aim(s)/objectives To ascertain the proportion of MSM attending our service eligible for PrEP on clinical grounds and estimate the service and cost implications of introducing PrEP.

Methods 97 MSM attending from December 2014 to July 2015 were reviewed, including patients who had attended for postexposure prophylaxis and STI screening. Patients were assessed against the PROUD study inclusion criteria to identify those who would benefit from PrEP.

Results

\begin{tabular}{|c|c|}
\hline Inclusion criteria & $\begin{array}{l}\text { Percentage of } \\
\text { patients who fit } \\
\text { the criteria }\end{array}$ \\
\hline Born male & $100 \%$ \\
\hline Completed HIV and STI screen & $84 \%$ \\
\hline HIV negative within previous 4 weeks & $95 \%$ \\
\hline $\begin{array}{l}\text { Unprotected anal intercourse (UPAI) on > one occasion } \\
\text { in the previous } 90 \text { days }\end{array}$ & $16 \%$ \\
\hline $\begin{array}{l}\text { All criteria fulfilled (Having attended at least once } \\
\text { previously was not considered as this was felt unnecessary) }\end{array}$ & $14 \%$ \\
\hline
\end{tabular}

No patients met any of the PROUD exclusion criteria. In a further $21 \%$ of notes, the exact number of episodes of UPAI, if any, was unclear although all other criteria were met. This suggests a range of $14-35 \%$ eligible for PrEP. We assumed all patients would continue having UPAI, were willing to comply with additional visits and were able to provide consent. The annual cost of Truvada is $£ 356$ per year. Further service cost analyses will be described.

Discussion/conclusion At least 14-35\% of MSM attending our service may benefit from PrEP provision. Additional costs include STI screening, monitoring and care pathways will need to be introduced. However, the overall impact of PrEP may be to reduce new HIV diagnoses and the resultant care costs. 